\title{
Origem anômala da artéria circunflexa da artéria pulmonar direita
}

\author{
José Carlos R. IGLÉZIAS*, Naora Miura IRAKI*, Carlos Augusto DIAS*, Luiz Alberto DALLAN*,
} Sérgio de Almeida OLIVEIRA*, Adib D. JATENE*

IGLÉZIAS, J. C. R.; IRAKI, N. M.; DIAS, C. A.; DALLAN, L. A.; OLIVEIRA, S. A.; JATENE, A. D. - Origem anómala da artéria circunflexa da artéria pulmonar direita. Rev. Bras. Cir. Cardiovasc., 4(2): 173-176, 1989.

RESUMO: É relatado, pela primeira vez na literatura, o caso de paciente adulto de 35 anos, com queixa de dor precordial aos esforços desde há 17 anos e que, após ter sido submetido a correçāo de coarctação de aorta, foi investigado e teve como diagnóstico: origem anómala da artéria circunflexa. No intra-operatório, constatou-se que a mesma tinha origem na artéria pulmonar direita. Foi realizada a sutura do óstio anómalo e um enxerto livre da artéria torácica interna direita para a coronária circunflexa. Além da raridade da lesăo, são possíveis consideraçōes fisiopatológicas relacionadas com a coarctaçăo da aorta, neste caso especifico.

DESCRITORES: artéria circunflexa, origem anômala.

\section{INTRODUÇÃO}

A origem anômala das artérias coronárias é uma cardiopatia congênita rara e grave. Cerca de $90 \%$ dos pacientes desenvolvem insuficiência cardíaca e morrem na infância ${ }^{6.9}$. Desde a revisão feita por $\mathrm{OGDEN}^{7} \mathrm{em}$ autopsias, foram relatadas algumas situaçōes anatômicas raras, nas quais a origem anômala da artéria coronária era da artéria pulmonar direita.

Não há, na literatura mundial, relato desta situação anatômica em paciente adulto.

\section{RELATO DE CASO}

Em fevereiro de 1988, um paciente de 35 anos, masculino, branco, de nível superior, procurou o Serviço com queixa de precordialgia aos esforços desde 1971, após ter sido submetido a operação para correção de coarctação da aorta. Referia que, até a época da cirurgia, apresentava apenas episódios esporádicos de cefaléia e dor nos membros inferiores e que o diagnóstico de coarctação da aorta foi realizado após constatação de hipertensão arterial em exame clínico de rotina. Desde 1971, procurou vários serviços médicos, sem melhora da sintomatologia, mesmo medicado com betabloqueadores e bloqueadores dos canais de cálcio.

Ao exame físico, encontrava-se em bom estado geral, eutrófico, com pressão arterial nos membros superiores de $120 / 70$ e nos membros inferiores de $120 \mathrm{mmHg}$. No tórax apresentava cicatriz inframamária à esquerda. A ausculta pulmonar era normal. No pericórdio, o ictus năo era palpável, as bulhas cardíacas eram rítmicas e havia discreta hiperfonese da segunda bulha na área aórtica. Apresentava sopro sistólico mais intenso em área mitral. No abdome, não apresentava alteraçōes e todos os pulsos eram palpáveis e normais.

Aos raios $X$ de tórax, apresentava área cardíaca normal e discreta reação pleural à esquerda (Figura 1).

Trabalho realizado no Instituto do Coração do Hospital das Clínicas da Faculdade de Medicina da Universidade de São Paulo. Sáo Paulo, SP, Brasil.

Recebido para publicação em 12 de agosto de 1989

- Do Instituto do Coração do Hospital das Clínicas da Faculdade de Medicina da Universidade de São Paulo.

Endereço para separatas: José Carlos Iglézias. Av. Dr. Enéas de Carvalho Aguiar, 44. Divisão Cirúrgica. 05403 Săo Paulo, SP, Brasil. 
IGLÉZIAS, J. C. R.; IRAKI, N. M.; DIAS, C. A.; DALLAN, L. A.; OLIVEIRA, S. A.; JATENE, A. D. - Origem anómala da artéria circunflexa da artéria pulmonar direita. Rev. Bras. Cir. Cardiovasc., 4(2): 173-176, 1989.

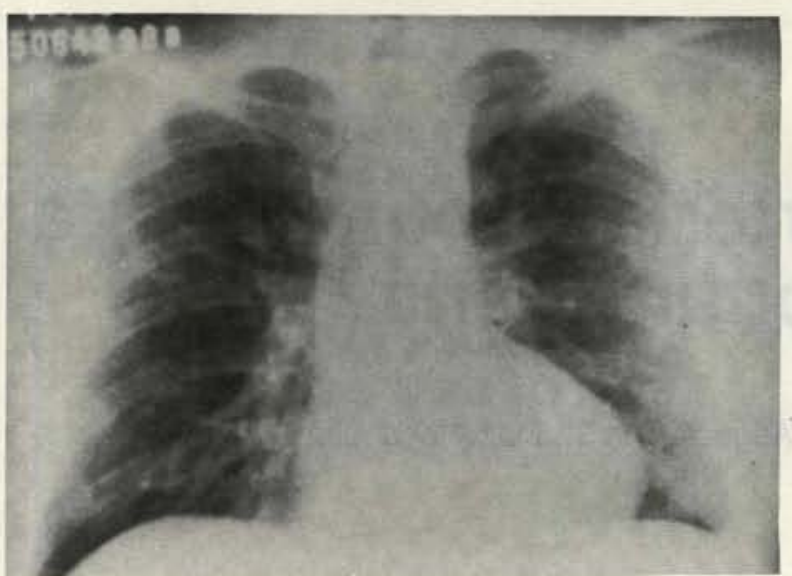

Fig. 1 - Radiografia de tórax pré-operatória: nota-se o espessamento pleural à esquerda, devido à correção da coarctaçâo da aorta que foi realizada por toracotomia esquerda.

O eletrocardiograma era normal. O ecocardiograma mostrava valva aórtica bivalvulada, com insuficiência discreta, arco aórtico sem estenoses ou dilataçōes, funçāo ventricular normal, fração de ejeção de $53 \%$ e relação volume/massa de 0,59 .

No teste ergométrico, o paciente atingiu freqüência cardiaca cardíaca máxima, mostrando infradesnivelamento do ponto $\mathrm{J}$ de $2 \mathrm{~mm}$ e segmento ST horizontal no décimo quinto minuto (Figura 2 ). Na cintilografia mio-

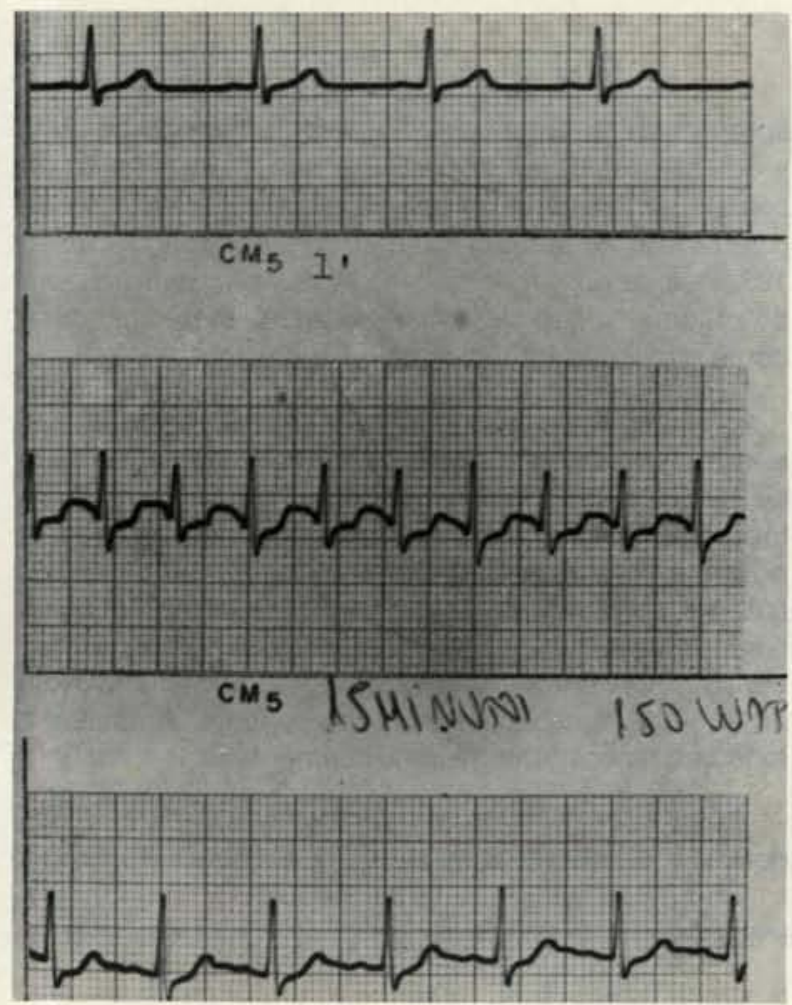

Fig. 2 - ECG de esforço evidenciando o infradesnivelamento do ponto J e segmento ST no 15 '. cárdica de esforço com tálio, foi diagnosticada isquemia miocárdica posterior do ventrículo esquerdo, sem redistribuição em repouso.

As medidas hemodinâmicas foram normais. A cinecoronariografia mostrava artéria circunflexa com origem anômala, que se enchia por circulaçāo colateral da artéria descendente anterior e da artéria coronária direita (Figura 3). Nāo foi possível determinar com precisão o local de origem da artéria circunflexa; a princípio, considerou-se como tendo origem na artéria pulmonar. A função ventricular na ventriculografia era normal e a correção da coarctação da aorta estava perfeita.

Em março de 1988, o paciente foi submetido a tratamento cirúrgico, por esternotomia mediana, com auxílio da circulação extracorpórea e hipotermia. Após a arteriotomia do tronco pulmonar, não foi possível evidenciar o óstio anômalo. Em seguida, a pinça da aorta foi aberta e, então, pudemos visibilizar um jato sangüíneo proveniente da artéria pulmonar direita. A arteriotomia foi prolongada até a artéria pulmonar direita, onde se localizava o óstio anômalo, que foi suturado (Figura 4). O paciente foi revascularizado com enxerto livre da artéria torácica interna direita para a coronária circunflexa, que nascia isoladamente, ou seja, separada da descendente anterior.

No pós-operatório imediato, não houve intercorrências e o paciente recebeu alta no sétimo dia de pós-operatório.

\section{DISCUSSÃO}

Cerca de $10 \%$ dos pacientes com origem anômala de artéria coronária permanecem vivos até a adolescência e a idade adulta ${ }^{6,9}$. Os pacientes que sobrevivem até essa idade o fazem devido ao desenvolvimento de

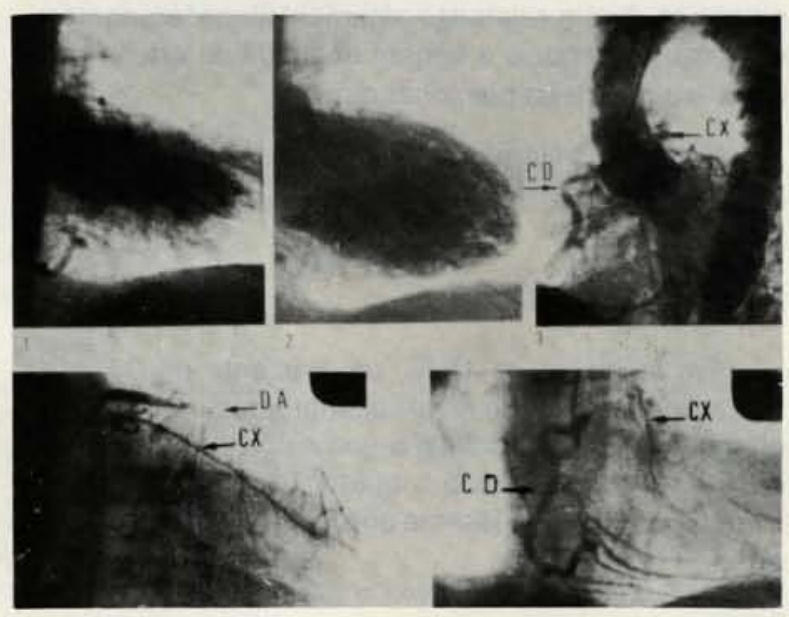

Fig. 3 - Detalhes do estudo hemodinâmico evidenciando o enchimento da artéria coronária circunflexa anómala a partir de circulaçāo colateral, provenientes da descendente anterior e coronária direita. 


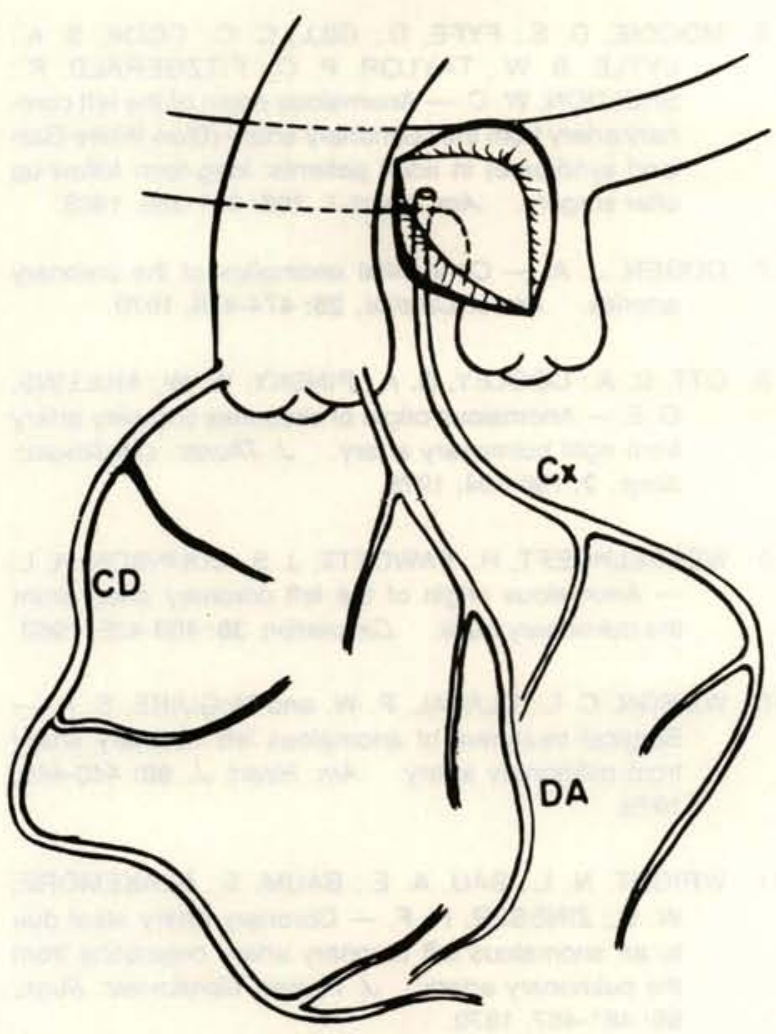

Fig. 4 - Representação esquemática da origem da artéria circunflexa (CX) na artéria pulmonar direita.

importante rede colateral da artéria coronária, que se origina da aorta e da importância da artéria anômala para a irrigaçāo da massa miocárdica. EDWARDS ${ }^{2}$ descreveu três fases clínicas desta anomalia quando ela permite a sobrevida até a idade adulta. A primeira fase acontece quando a pressāo pulmonar ainda é relativamente alta no recém-nato, permitindo uma adequada perfusão através da artéria de origem anômala e a criança aparenta normalidade ao nascimento e até algum tempo após. A segunda fase caracteriza-se pela queda dos niveis pressóricos no sistema pulmonar, e a perfusão miocárdica pode não acontecer adequadamente. Nesta fase, a sobrevivência dependerá do desenvolvimento da circulaçăo colateral e poderá ocorrer isquemia miocárdica, insuficiência cardíaca e arritmias, levando à morte. A terceira fase existirá se o paciente tiver oportunidade de desenvolver circulação colateral e sobreviver até a adolescência ou a idade adulta. Nesta fase, podem ocorrer arritmias, morte súbita e insuficiência mitral pelo aumento da área cardíaca.

WRIGHT et alii ${ }^{11}$ propōem uma quarta fase quando o paciente atinge a idade adulta, qual seja a fase do roubo do fluxo. A formação de intensa rede de colaterais que coleta o sangue vindo da aorta para um sistema de drenagem na artéria pulmonar diminui a pressāo intracoronariana e prejudica a perfusão miocárdica, possibilitando, assim, manifestaçōes isquêmicas. No presente caso, o roubo de fluxo é evidente. O paciente era portador de duas lesões: coarctação da aorta e origem anômala da artéria circunflexa. A coarctação fazia com que a pressão na raiz da aorta fosse bem mais alta que o habitual, aumentando a pressão de perfusão do miocárdio. Enquanto o paciente não fora submetido a correção de coarctação, não apresentava manifestações da doença isquêmica do miocárdio. Após a correçăo da coarctação, com a normalização das pressōes na porção ascendente da aorta, o paciente passou a apresentar precordialgia devido a baixa pressāo de perfusāo pelo sistema coronariano colateral. Por um determinado período da vida, o miocárdio permaneceu "protegido" pela coarctaçāo da aorta.

Quando existe uma circulação colateral bem desenvolvida entre as artérias coronárias, apenas a ligadura do óstio anômalo é suficiente para melhorar a pressão de perfusão intracoronariana ${ }^{1}, 3,11$. No entanto, WILSON et alii ${ }^{10}$ sugerem que, do ponto de vista da evolução tardia, os resultados seriam mais satisfatórios quando é feita a revascularização da artéria com origem anômala após a sutura do óstio, ou a transposição da mesma para a aorta.

RBCCV 44205-90

IGLÉZIAS, J. C. R.; IKARI, N. M.; DIAS, E. A.; DALLAN, L. A.; OLIVEIRA, S. A.; JATENE, A. D. - Anomalous origin of circunflex artery from the right pulmonary artery. Rev. Bras. Cir. Cardiovasc., 4(2): 173-176, 1989.

ABSTRACT: A case never described in adults before is here reported. A 35-year-old patient who had referred precordial pain in effort for seventeen years was investigated after having been submitted to correction of coarctation of the aorta, and had the following diagnosis: anomalous origin of the circunflex artery. In the intraoperatory, the latter was found to proceeed from the right pulmonary artery. The anomalous ostio was sutured and a free graft was made, from the right internal thoracic artery to the circunflex coronary. In addition to the unusualness of such pathology, some physiopatologic considerations related to the coarctation of the aorta may be made, in this particular case.

DESCRIPTORS: circunflex artery, anomalous origin. 
IGLÉZIAS, J. C. R.; IRAKI, N. M.; DIAS, C. A.; DALLAN, L. A.; OLIVEIRA, S. A.; JATENE, A. D. - Origem anómala da artéria circunflexa da artéria pulmonar direita. Rev. Bras. Cir. Cardiovasc., 4(2): 173-176, 1989.

\section{REFERÊNCIAS BIBLIOGRÁFICAS}

1 ARCINIEGAS, E.; FAROOKI, Z. Q.; HAPIM, M.; GREEN, E. W. - Management of anomalous left coronary artery from the pulmonary artery. Circulation, 62: 180-188, 1980.

2 EDWARDS, J. E. - The direction of blood flow in coronary arteries arising from the pulmonaryc trunk. Circulation, 29: 163-166, 1964.

3 FLAMM, M. D.; STENSON, E. B.; HULTGREEN, H. N.; SHUMWAY, N. E.; HANCOCK, E. W. - Anomalous origin of the left coronary artery from the pulmonary artery: surgical treatment by ostial occlusion through pulmonary arteriotomy. Circulation, 38: 113-123, 1968.

4 HAMILTON, J. R. L.; MULHOLLAND, H. C.; O'KANE, H. O. J. - Origin of the left coronary artery from the right pulmonary artery: A report of successful surgery in a 3-month-old child. Ann. Thorac. Surg., 44: 446-448, 1986.

5 HONEY, M.; LINCOLN, J. C. R.; OSBORNE, M. P.; DeBONO, D. P. - Coarctation of the aorta with right aortic arch. Report of surgical correction in 2 cases: One with associated anomalous origin of left circunflex coronary artery from the right pulmonary artery. Br. Heart. J., 37: 937-945, 1975.
6 MOODIE, D. S.; FYFE, D.; GILL, C. C.; COOK, S. A.; LYTLE, B. W.; TAYLOR, P. C.; FITZGERALD, R.; SHELDON, W. C. - Anomalous origin of the left coronary artery from the pulmonary artery (Blan-White-Garland syndrome) in adult patients: long-term follow-up after surgery. Am. Heart J., 106: 381-388, 1983.

7 ODGEN, J. A. - Congenital anomalies of the coronary arteries. Am. J. Cardiol., 25: 474-479, 1970.

8 OTT, D. A.; COOLEY, D. A.; PINSKY, W. W.; MULLINS, C. E. - Anomalous origin of circunflex coronary artery from right pulmonary artery. J. Thorac. Cardiovasc. Surg., 2: 190-194, 1978.

9 WESSELHOEFT, H.; FAWCETT, J. S.; JOHNSON, A. L. - Anomalous origin of the left coronary artery from the pulmonary trunk. Circulation, 38: 403-425, 1968.

10 WILSON, C. L.; DLABAL, P. W. and McGUIRE, S. A. Surgical treatment of anomalous left coronary artery from pulmonary artery. Am. Heart. J., 98: 440-446, 1979.

11 WRIGHT, N. L.; BAU, A. E.; BAUM, S.; BLAKEMORE, W. S.; ZINSSER, H. F. - Coronary artery steal due to an anomalous left coronary artery originating from the pulmonary artery. J. Thorac. Cardiovasc. Surg., 59: $461-467,1970$. 